\title{
Impact of Mood Changes on Application Selection
}

$\begin{array}{ll}\begin{array}{l}\text { Aku Visuri } \\ \text { University of Oulu } \\ \text { akuvisur@ee.oulu.fi }\end{array} & \begin{array}{l}\text { Evangelos Karapanos } \\ \text { Cyprus University of Technology } \\ \text { evangelos.karapanos@cut.ac.cy }\end{array} \\ \begin{array}{l}\text { Zhanna Sarsenbayeva } \\ \text { University of Oulu }\end{array} & \begin{array}{l}\text { Simon Jones } \\ \text { University of Bath } \\ \text { zsarsenb@ee.oulu.fi }\end{array} \\ \begin{array}{l}\text { Jorge Goncalves } \\ \text { University of Oulu } \\ \text { jgoncalv@ee.oulu.fi }\end{array} & \end{array}$

\section{Abstract}

Users of quantified self applications habitually log and track personal information, such as mood. Attempts to automate the procedure of logging mood have been made, but applications themselves rarely provide insights into the user's mental well-being. In this paper we explore data from two small scale studies related to mobile device usage and mood tracking. We analyse associations between user's mood throughout the day and the use of smartphone applications from different

categories. Our analysis provides insights into the user's behaviour based on their device usage. These insights mean that QS applications can independently use simple analysis tools to provide similar insights for the user.

\section{Author Keywords}

Mood-sensing; behaviour; application usage

\section{ACM Classification Keywords}

H.5.m. Information interfaces and presentation (e.g.,

$\mathrm{HCI}$ ): Miscellaneous; 


\section{Communication \\ Communication}

\section{Productivity and Admin Productivity \\ Tools \\ Education \\ Business \\ Books and Reference \\ Finance \\ Personalization}

\section{Health}

Lifestyle

Health and Fitness

\section{Internet \& Social Media}

Entertainment

News and Magazines

Sports

Social

\section{Maps and Travel \\ Travel and Local \\ Weather}

\section{Media}

Music and Audio

Photography

Media and Video

\section{Games}

Different game genres

(strategy, puzzle, etc.)

\section{Other}

Applications not listed in Google PlayStore

Table 1: Application category grouping.

\section{Introduction}

Many individuals employ Quantified Self (QS) applications nowadays to uncover insights around their behaviors and feelings. The insights that users gain from these applications, however, are usually selfperceived, as either the application's intelligence or the amount of information gathered from the application is too limited to automatically draw definitive insights for the user. This paper aims to analyse the feasibility of programmatically gaining insights beneficial to the user from the gathered information of the collective user population as well as the individual user.

Previous research conducted by the authors and their collaborators has involved running small scale studies to gather sensor data from mobile devices, as well as self-reported data from the study participants. This paper aims to analyse two of these of existing datasets to search for associations between reported and sensed factors.

We draw upon data from two different field studies, aggregating self-reported mood information, sensor based collection of application usage, and the periods of day when this data was logged. We then analyse the correlations between the mood and application usage and present our findings in terms of how the reported values from these studies performed in showcasing behavioral patterns for the users and how this could influence application design.

\section{Related Work}

As mental well-being plays a profound role in people's health and quality of life [4], a wide range of research studies have attempted to study people's sentiment based on their use of mobile devices, for instance by looking at phone usage and contextual data [2], social interaction [6], and sleep and physical activity [10]. An underexplored predictor of individual's mental wellbeing is application usage. Niforatos et al. [8] found the use of certain types of mobile applications to correlate with heightened levels of mental wellbeing, while Lin et al. [7] found that social media use among young adults to have a significant association with depression.

QS applications generally suffer from lack of user engagement and motivation $[1,3,9]$, to which,

Crowdwalk, for example, offers a solution by providing insights about the user and his or her actions. We suggest that QS applications can perform automated analyses, seeking for significant correlations among users' behaviors, contextual factors, and psychological states, and thus lowering the motivational demands for the user. To this end, personal mobile devices, due to their frequent use, exhibit potential to become effective life companions that can track the emotional state of the user and provide advice or further assistance, for instance when inferring a social anxiety event or rapid deterioration of mental well-being.

\section{Datasets}

From two independent field studies we obtained data on mobile device sensors and user reported data using the Experience Sampling Method [5]. Each study is analysed separately to maintain the internal validity. Studies are referred to as Study A and B henceforth. Study settings are described in Table 2 . Study participants were students or employees of our university, roughly half men, half women, aged between 20 and 40 . 


\begin{tabular}{c|lll} 
Study & Duration & $\begin{array}{l}\text { \# of } \\
\text { users }\end{array}$ & $\begin{array}{l}\text { Tracked } \\
\text { Data }\end{array}$ \\
\hline A & 2 weeks & 15 & H, Ap \\
B & 2 weeks & 21 & P, Act, Ap
\end{tabular}

Table 2: Study details; $\mathrm{H}=$ Happiness, $\mathrm{P}=$ Pleasure, Act $=$ Activeness, $\mathrm{Ap}=$ Application Usage

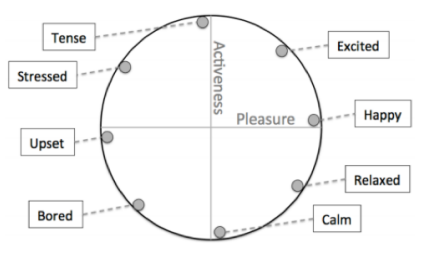

Figure 1: The circumplex model of affect model.
In Study A we gathered controlled ESM responses which measured individuals' happiness on a Likert scale. In Study B each user reports pleasure and activeness levels according to the circumplex mood model [11] (Figure 1), via an ESM with Likert scales for both variables. We use the sensor capabilities of each device to log the changes in foreground applications. Similar to Brown et al. [1], we classified applications using the PlayStore application category scheme as described in Table 1.

The datasets were preprocessed to reduce the amount of outliers and possibly incorrect information. For Study $B$, we only considered ESM responses with an interval of at least 30 minutes in order to reduce duplicate values, and minimize negligently inputted values due to the burden that the ESM method placed on the users. For application use in terms of reported mood, we consider only the applications launched in a five minute time window after the ESM response. This means that our analysis is likely to capture what applications were launched while experiencing a certain mood. All inputted data across studies is normalised to use the same scale ('Low', 'Neutral', 'High') for each variable.

\section{Results}

We analyse the data gathered from both studies individually to see how mood, application usage, and time of the day interact with each other. This enables us to create a more detailed view of each user's device usage behavior, and test general assumptions of application usage and mood patterns. We first analyse the gathered mood and then analyse each individual user separately in terms of cross-comparing the three tracked variables (time, mood, application usage) for both studies A and B.
The raw datasets include a total of 35025 instances of foreground application changes ( $M=972$ per user) and 9171 ( $M=254$ per user) entries of self-reported mood information.

Mood

Our two studies tracked either activeness and pleasure values (Study B) or just users' happiness (Study A). According to the circumplex model of affect, happy is a mood category with a neutral activeness value, and the highest pleasure value, as shown in Figure 1, and upset is the opposite end of the pleasure axis. We use the reported pleasure value from Study $B$ as a surrogate for the happiness values from Study A when comparing the analysis results between studies.

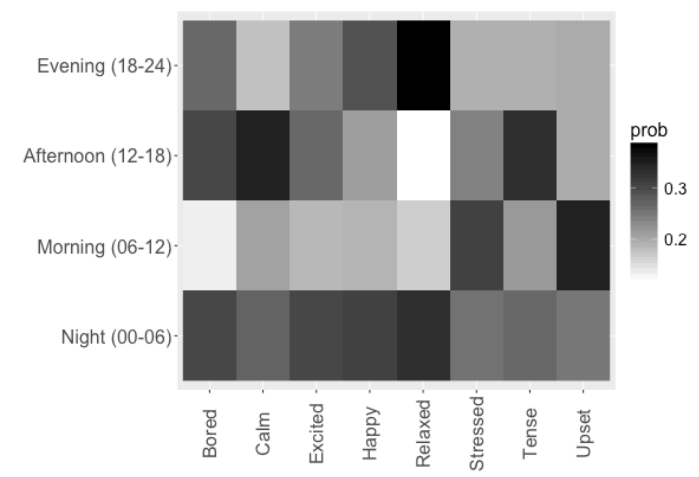

Figure 2: Study B mood categories across periods of day.

We first use Pearson's product moment correlation to test the relationship between the reported activeness and pleasure values and the time of the day. We observe significant changes for both the activeness and pleasure levels over the course of the day using the chi-square test of independence between the hour of 

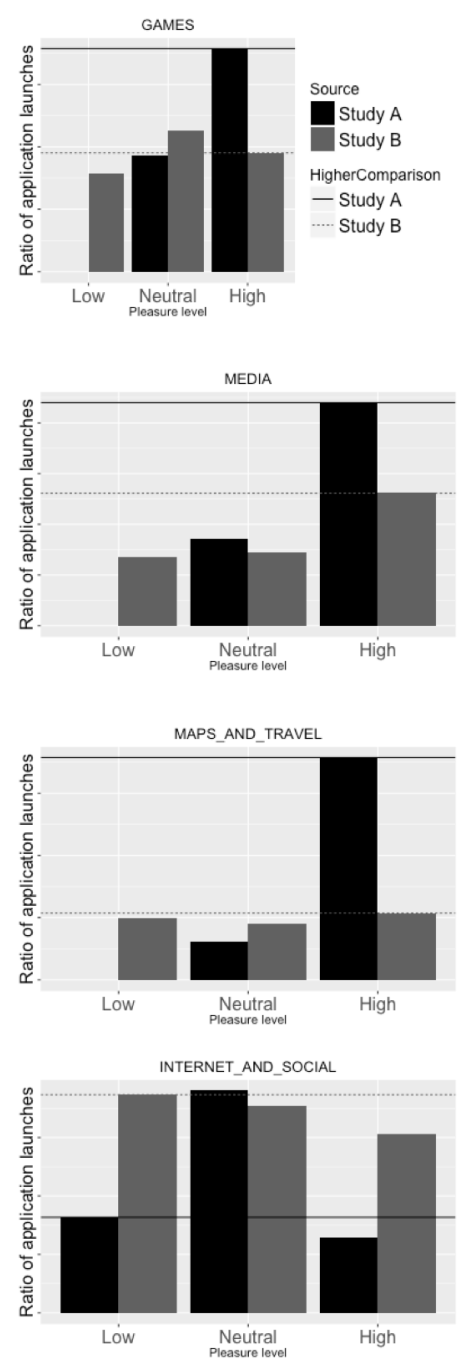

Figure 4: Application usage between pleasure levels in studies $A$ and $B$ - lines aid in comparison between low and high levels. the day and the reported mood values, for both Study $\mathrm{A}\left(x^{2}(602)=944, p<0.05\right)$ and Study $\mathrm{B}\left(x^{2}(3780)=\right.$ $5508, p<0.05)$.

Additionally, we categorise the participants' mood from Study B in terms of the different mood categories presented in Figure 1 by using reported values from each ESM response and mapping the values in the circumplex model of affect. The mood categories are happy, excited, tense, stressed, upset, bored, calm, and relaxed. $35.2 \%$ of the instances were classified as happy, and $20.1 \%$ as excited, combining for $55.3 \%$ of all reported moods. A chi-square test reveals a significant association between mood and period of day (Night, Morning, Afternoon, Evening; $X^{2}(21)=168.2, p$ $<.05)$. The distribution of mood experienced in different periods of day is shown in Figure 2.

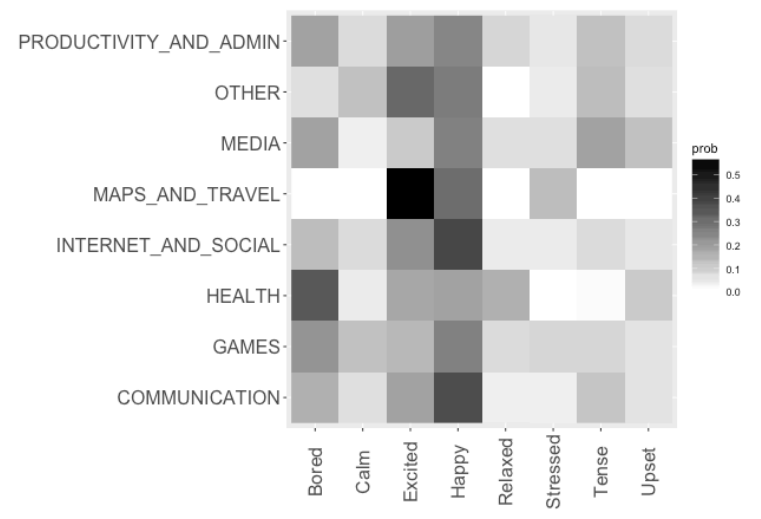

Figure 3: Study B application categories used in different mood categories.

\section{Application Categories}

To analyse how mood levels and categories correspond to application usage for different application categories, we use chi-square tests to infer the association between mood levels and used application categories. In both Studies $A$ and $B$, the happiness level and the used application categories were significantly related $\left(X^{2}(14)=38.61, p<0.05\right)$, and in Study $B$ both the activeness level $\left(X^{2}(14)=84.59, p<0.05\right)$ and happiness level $\left(x^{2}(14)=32.90, p<0.05\right)$ were significantly related with the used application categories. Additionally, using chi-squared test we revealed a semi-weak (Cramer's $\mathrm{V}=.23$ ) relation between mood category and application category ( $x$ $\left.{ }^{2}(49)=328.29, p<.05\right)$. Results are visualised in Figure 3. The application launch distribution of applications categories where users showed similar differences between low and high pleasure levels in both studies are visualised in Figure 4.

\section{Independent Users}

Finally we analyse each user $(n=22)$, with at least 20 self-reported entries, independently of others using the chi-square test between mood levels and period of day or application usage. Number of users who have either a) significant association between activeness and application category, b) significant association between pleasure and application category c) sig. assoc. between time of day and pleasure, d) sig. assoc. between time of day and activeness is $\mathbf{1 6}$ out of $\mathbf{3 6}$. $50 \%$ of the significant tests are associations between time of day and pleasure levels, $32.1 \%$ between activeness and time of day, $10.7 \%$ between activeness and application category, and $7.1 \%$ between pleasure and application category. 


\section{Discussion}

Analysing our gathered data sets from a relatively short time period, we find several associations in the general population of our studies as well as for individual participants.

All in all, we found user's mood to vary systematically over the course of the day (see Figure 2). People generally feel upset or stressed in the morning hours, calm or tense in the afternoon and relaxed in the evening. Study B further highlighted the applications that are more likely to be used in certain mood (see Figure 3). People are most likely, for instance, to use Maps and Travel applications when feeling excited, Health or Productivity, Admin when bored, and Media when either tense or bored. Due to the large sampling $(55.3 \%)$ of mood categories being either excited or happy, most applications were most likely to be used in these two states.

We also identified associations between application usage and the different pleasure levels (study B) or happiness levels (study A) experienced by user (see Figure 4). Some similarities existed across both studies especially when comparing low to high pleasure levels - namely in the use of applications in Media, Games, Maps and Travel (more likely to use when happy), and Internet and Social (slightly more likely to use when unhappy). Our result regarding social media use associated with negative moods are also similar with the results of Lin et al. [6] and their studies of social media use in large scale, albeit in a different causality direction.

From analysing users independently, it is evident that the strongest impact on mood and device usage on a personal level derives from the time of day as $82.1 \%$ of the statistically significant results for individual users were associations between time and either application usage or mood levels.

These findings indicate that insights into user's behaviour can be generated using simple analysis tools. The associations between the period of the day, the user's mood levels, and application usage for both general populations and on a user level may be used both to provide insight to the user and to enhance application usability. The need for manual input for selfreported values, such as mood, may be overcome using assumptions based on the general population, or the application usage logs of the user. These assumptions may also be correlated to changes in application preferences or application functionality, e.g. when to send reminders to users, or when are users more likely to use specific applications. Applications relying on mood self-reporting can also attempt to predict and pre-select self-reports based on the used applications.

\section{Conclusion}

We analyse self-reported mood information and application usage from two small scale studies, and find significant correlations between the reported mood states, periods of day, and application usage. According to our results, people tend to use Media, Games, Maps, and Travel applications when their pleasure level is higher, and tend to use Internet and Social apps when their pleasure level is lower. People are also likely to use different types of applications in different mood states. These findings allow insights about the end user to be drawn from personally collected datasets, partly removing the need for modeling large 
populations, and enhancing the potential to adapt applications with these predictions.

\section{Acknowledgements}

This work is partially funded by the Academy of Finland (Grants 276786-AWARE, 285062-iCYCLE, 286386CPDSS, 285459-iSCIENCE), and the European Commission (Grants PCIG11-GA-2012-322138, 645706-GRAGE, and 6AIKA-A71143-AKAI)

\section{References}

1. Barry Brown, Moira McGregor and Donald McMillan. 2014. 100 Days of iPhone Use: Understanding the Details of Mobile Device Use. In Proceedings of the 16th International Conference on Human-computer Interaction with Mobile Devices \& Services, ACM, 223 232. http://dx.doi.org/10.1145/2628363.2628377 2. Michelle M. N. Burns, Mark Begale, Jennifer Duffecy, Darren Gergle, Chris C. J. Karr, Emily Giangrande and David D. C. Mohr. 2011. Harnessing Context Sensing to Develop a Mobile Intervention for Depression. Journal of Medical Internet Research 13, 3. http://dx.doi.org/10.2196/jmir.1838

3. Rúben Gouveia, Evangelos Karapanos and Marc Hassenzahl. 2015. How Do We Engage with Activity Trackers?: A Longitudinal Study of Habito. In Proceedings of the 2015 ACM International Joint Conference on Pervasive and Ubiquitous Computing, ACM, 1305-1316.

http://dx.doi.org/10.1145/2750858.2804290

4. Aron Halfin. 2007. Depression: the benefits of early and appropriate treatment. The American journal of managed care 13, 4 Suppl: S92-7.

5. Joel J. M. Hektner, Jennifer J. A. Schmidt and Mihaly Csikszentmihalyi. 2007. Experience Sampling
Method: Measuring the Quality of Everyday Life. Sage. 6. Michael M. D. Lee, Mark Steyvers, Mindy De Young and Brent Miller. 2012. Inferring expertise in knowledge and prediction ranking tasks. Topics in cognitive science $4,1: 151-163$.

7. Liu L. Y. Lin, Jaime J. E. Sidani, Ariel Shensa, Ana Radovic, Elizabeth Miller, Jason J. B. Colditz, Beth B. L. Hoffman, Leila L. M. Giles and Brian B. A. Primack. 2016. ASSOCIATION BETWEEN SOCIAL MEDIA USE AND DEPRESSION AMONG U.S. YOUNG ADULTS. Depress Anxiety 33, 4: 323-31. http://dx.doi.org/10.1002/da.22466

8. Evangelos Niforatos and Evangelos Karapanos 2014. EmoSnaps: a mobile application for emotion recall from facial expressions. Personal and Ubiquitous Computing 19, 2: 425-444.

http://dx.doi.org/10.1007/s00779-014-0777-0

9. Tiago Ornelas, Ana Caraban, Rúben Gouveia and Evangelos Karapanos. 2015. CrowdWalk: leveraging the wisdom of the crowd to inspire walking activities. In Proceedings of the 2015 ACM International Joint Conference on Pervasive and Ubiquitous Computing and Proceedings of the 2015 ACM

International Symposium on Wearable Computers, 213216.

10. Mashfiqui Rabbi, Shahid Ali, Tanzeem

Choudhury and Ethan Berke. 2011. Passive and In-Situ Assessment of Mental and Physical Well-being Using Mobile Sensors. In Proceedings of the 13th

International Conference on Ubiquitous Computing, ACM, 385-394.

http://dx.doi.org/10.1145/2030112.2030164

11. James J. A. Russell. 1980. A circumplex model of affect. Journal of Personality and Social Psychology 39, 6: 1161. http://dx.doi.org/10.1037/h007771 